\title{
Synovial fibroblast hyperplasia correlates with rheumatoid arthritis duration and activity and is partially reversed by anti-TNF therapy
}

\author{
E Izquierdo ${ }^{1 *}$, J D Cañete ${ }^{2}$, R Celis ${ }^{2}$, M J Del Rey ${ }^{1}$, A Usategui ${ }^{1}$, S Marsal ${ }^{3}$, R Sanmartí ${ }^{2}$, G Criado $^{1}$, J L Pablos
}

From 5th European Workshop on Immune-Mediated Inflammatory Diseases

Sitges-Barcelona, Spain. 1-3 December 2010

\section{Introduction}

Synovial fibroblasts (SF) hyperplasia has been proposed to contribute to the pathogenesis of rheumatoid arthritis (RA). Information on the dynamics of SF in RA is scanty since no reliable quantitative markers are available.

\section{Aim}

To evaluate immunolabeling of the fibroblast-specific chaperon hsp47 as a quantitative marker for SF and to analyse its clinicopathological correlates and evolution after anti-TNF $\alpha$ therapy.

\section{Patients and methods}

Synovial arthroscopic biopsies were obtained from RA $(n=48)$ and osteoarthritis $(O A ; n=9)$ patients and normal controls $(n=6) .25$ patients with active disease were rebiopsied after $10 \pm 2$ months of anti-TNF- $\alpha$ therapy. Immunoperoxidase or immunofluorescent labeling for hsp 47 (cloneM16.10A1), CD68, CD3, CD20, CD31 and alphasmooth muscle actin (aSMA) was performed. Hsp47-positive fractional area in the lining or sublining was quantified by ImageJ software. Correlation with clinicopathological variables was analyzed by Pearsons or Spearman's test where appropriate. Changes after anti-TNF therapy were analysed with Student's $t$-test for paired data.

\section{Results}

In all healthy or diseased synovial tissues, anti-hsp47 immunolabeling specifically identified fibroblastic cells of the lining and sublining, and perivascular fibroblasts or pericytes.

Mononuclear cells, vascular CD31-positive endothelial cells and smooth muscle cells were not labelled. Lining SF

${ }^{1}$ Servicio de Reumatología, Hospital 12 de Octubre, Instituto de Investigación Hospital 12 de Octubre (I+12), Madrid, Spain

Full list of author information is available at the end of the article area was significantly increased in RA and OA tissues compared to healthy tissues. In RA, a significant increase of the sublining SF area compared to either OA or healthy tissues was found. Lining SF area was significantly and positively correlated with the density of CD68-positive macrophages, DAS28, and disease duration. In contrast, the increase in the area of sublining SF was negatively correlated with disease duration and DAS28. A significant reduction of lining but not sublining SF area was observed after anti-TNF therapy. A higher but no statistically significantly different reduction of the lining SF area was observed in the group of EULAR responders.

\section{Conclusion}

Hsp47 is a specific marker of SF that permits to obtain quantitative data from human synovial tissues. Our data suggest that lining but not sublining SF expansion is a dynamic component of RA synovitis that parallels inflammatory activity and progression of the disease, and that can be partially reversed by anti-TNF therapy.

\section{Author details}

${ }^{1}$ Servicio de Reumatología, Hospital 12 de Octubre, Instituto de Investigación Hospital 12 de Octubre (I+12), Madrid, Spain. ${ }^{2}$ Unitat d'Artritis, Servei de Reumatologia, Hospital Clínic de Barcelona, IDIBAPS, Barcelona, Spain. ${ }^{3}$ Unitat de Reumatologia i Grup de Recerca de Reumatologia. Institut de Recerca, Hospital Vall d'Hebron, Barcelona, Spain.

Published: 25 November 2010

doi:10.1186/1479-5876-8-S1-P30

Cite this article as: Izquierdo et al:: Synovial fibroblast hyperplasia correlates with rheumatoid arthritis duration and activity and is partially reversed by anti-TNF therapy. Journal of Translational Medicine 2010 8(Suppl 1):P30. 